Please quote as: Klendauer, R.; Hoffmann, A.; Leimeister, J. M.; Berkovich, M. \& Krcmar, H. (2012): Using the IDEAL Software Process Improvement Model for the Implementation of Automotive SPICE. In: 5th International Workshop on Cooperative and Human Aspects of Software Engineering (CHASE), ICSE 2012 Workshop, Zurich, Switzerland. 


\section{Using the IDEAL Software Process Improvement Model for the Implementation of Automotive SPICE}

\author{
Ruth Klendauer \\ Advanced Engineering \\ SMR Automotive Mirrors Stuttgart GmbH \\ Stuttgart, Germany \\ ruth.klendauer@smr-automotive.com
}

\author{
Axel Hoffmann, \\ Jan Marco Leimeister \\ Information Systems \\ Kassel University \\ Kassel, Germany \\ \{axel.hoffmann; leimester\}@uni-kassel.de
}

\author{
Marina Berkovich, \\ Helmut Krcmar \\ Information Systems \\ Technische Universität München \\ Munich, Germany \\ \{berkovich;krcmar\}@in.tum.de
}

\begin{abstract}
Most suppliers in the automotive industry are facing the challenge of implementing Automotive SPICE, a domain-specific model of ISO/IEC 15504, which was first published in 2005. Original equipment manufacturers have increasingly requested SPICE certifications from their suppliers in order to effectively evaluate the development processes and identify reliable partners. To support organizations in their software process improvement effort, the Software Engineering Institute (SEI) developed the IDEAL model. This guide is, however, influenced by the SEI's work on CMM and based on the experiences with very large organizations. The goal of this paper is to examine the suitability of the IDEAL model for the implementation of Automotive SPICE at a medium-sized R\&D department. Preliminary results from an action research study at a global systems engineering company are discussed. The data indicate that adjustments are especially needed with regard to the factors participation and communication. Also, informal networks between companies, early decision-making and usability are shown to play an important role and might be added to the model.
\end{abstract}

Keywords-Automotive-SPICE; IDEAL-Model; software process improvement; improvement models

\section{INTRODUCTION}

Nowadays, due to the competitive pressure, the product life cycles in the automotive industry get shorter, whilst the products get more complex. More than $85 \%$ of a car's functionality is controlled by embedded software [1]. The ability to control the electronic and software functionality has become a crucial challenge for manufacturers, especially suppliers.

As the OEMs (original equipment manufacturers) focus on core competencies to develop the car as a whole, a huge part of their development work is being outsourced. With sourcing out part of their development and production, they do not have direct control over quality, costs and schedule anymore. Of particular importance are quality issues that customers associate with the OEMs, not with their suppliers. Thus, the OEMs need to find high quality producing and reliable suppliers. To determine the capability of the suppliers, automotive manufacturers started an initiative: Automotive SPICE (A-SPICE), a domain-specific model of
ISO/IEC 15504. Due to the increased software development work, suppliers have to redesign their processes in order to cope with the increased complexity and to ensure reliable processes and products.

Many companies fail while implementing software process improvement (SPI). Out of 1.638 organizations, only 34 percent proceeded to a second assessment, out of those that proceeded, 13 percent did not improve their capability to develop quality software and some even moved to a lower level of capability (3.1 percent) [2]. To support organizations in their software process improvement effort, the Software Engineering Institute (SEI) developed the IDEAL model as a guide [5].

The IDEAL model is, however, based on the experiences with large organizations and influenced by the SEI's work on CMM. Previous research shows that it has to be tailored and adjusted to a company, considering business objectives and resources. Unfortunately, there are no experiences of using the IDEAL model for A-SPICE reported in the literature.

The goal of this paper is to examine the suitability of the IDEAL model for the implementation of A-SPICE. We studied the use of IDEAL for A-SPICE in its organizational context through collaborative practice research (CPR), a particular form of action research [3]. We conducted the empirical study at SMR, a global tier 1 supplier in the automotive industry with headquarters in Germany. In this paper we discuss preliminary results from the first six months of collaboration.

The remainder of the paper is organized as follows. First of all, we give an overview of Automotive Spice and the IDEAL model. Next, we summarize the related work about implementing SPI with the IDEAL model. After a description of the research design in section 5 we present the results of the study. This is followed by the discussion.

\section{Automotive SPICE}

For software development in the automotive industry, a new standard was appointed in 2005: Automotive SPICE, which was derived from ISO/IEC 15504 (SPICE: "Software Process Improvement and Capability dEtermination") by AUTOSIG (Automotive Special Interest Group). A-SPICE differs from ISO/IEC 15504 in that it focuses on embedded software and loses the sight of software for PCs and client/server systems [1]. 
A-SPICE is an approach to reduce development time and costs whilst the product quality stays high [1]. It represents a standard assessment model for software development processes in the automobile industry and is accepted - even suggested - by the OEM software initiative HIS (Herstellerinitiative Software). Partners of the HIS are: AUDI AG, BMW Group, Porsche AG, Volkswagen AG [4].

As the 31 A-SPICE processes are very laborious, the HIS appointed the HIS-Scope, which limits assessments to the following 15 processes.

\section{A. Engineering Process Group}

The Engineering Process Group contains 9 processes:

ENG.2

ENG.3

ENG.4 System requirements analysis

ENG.5 System architectural design

ENG.6 Software requirements analysis

ENG.7

ENG.8

ENG.9

Software design

Software construction

Software integration test

Software testing

ENG.10

System integration test

System testing

\section{B. Supporting Process Group}

The Supporting Process Group consists of the following 4 processes:

SUP.1 Quality assurance

SUP.8 Configuration management

SUP.9 Problem resolution management

SUP.10 Change request management

\section{Management Process Group}

The Management Process Group contains of:

MAN.3 Project management

\section{Acquisition Process Group}

The Acquisition Process Group is an optional process:

ACQ.4 Supplier monitoring

In contrast to $\mathrm{CMM}(\mathrm{I})$, all these 15 process areas are already assessed at the basic level (A-SPICE level 1) and do not increase in number. However, the degree of complexity of each process increases with each capability level. Furthermore, A-SPICE focuses more on the technical engineering aspects than $\mathrm{CMM}(\mathrm{I})$.

\section{THE IDEAL MODEL}

The IDEAL model is a software process improvement (SPI) model, published in 1996 by the Software Engineering Institute (SEI) of Carnegie Mellon University [5]. The goal of SPI is to improve the development process of software. The IDEAL guidelines are for those organizations that initiate a SPI for the first time and even for those that are continuing an already established SPI [5].

The IDEAL model contains five phases with a generic description of a sequence of recommended steps. Many activities can be pursued simultaneously. "In practice the boundaries between the phases of IDEAL are not as clearly defined as shown in the model" $[5, \mathrm{p} .1]$. As can be seen in
Figure 1, it is a cycle, what makes it a repetitive process. In the next section, we will give a short description of the several phases.

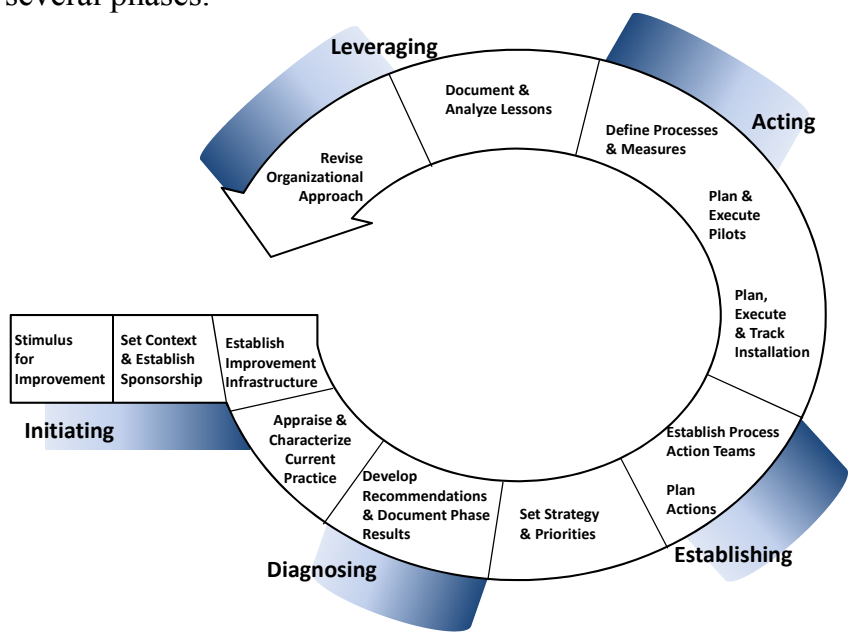

Figure 1. The IDEAL Model, following [5]

\section{A. Initiating Phase}

The Initiating Phase is the origin of the IDEAL model. The initial improvement infrastructure is established, the roles and responsibilities for the infrastructure are initially defined, and initial resources are assigned [5]. The aims for the SPI program are specified, the readiness of the organization is assessed and a SPI plan is created. For the supporting and the assisting infrastructure, it is typical to establish two key elements: a Management Steering Group (MSG) and a Software Engineering Process Group (SEPG) [5]. In summary, the first phase is for laying the groundwork [6].

\section{B. Diagnosing Phase}

In conformity with the organization's vision, the strategic business plan, lessons learned from past improvement efforts and other strategic factors, the SPI action plan is initiated. Evaluations are accomplished to get up-to-date data of the organization's state. These results are compared with concluded and planned process improvements. The findings are included in the SPI action plan [5]. Thus the Diagnosing Phase is for determining the status quo and the future goals [6].

\section{Establishing Phase}

The Establishing Phase is where the goals of the process improvement effort are prioritized and the approaches are developed. Also, the SPI action plan, which was initiated in the Diagnosing Phase, will be completed. This includes deriving measurable goals from the main goals. Training for the technical working groups (TWGs) is organized and resources are furnished. Also, action timelines are created and handed over to the TGWs [5]. In the Establishing Phase it is planned how the goals shall be achieved [6]. 


\section{Acting Phase}

In the Acting Phase solutions for improvement are created, piloted and then deployed in the entire organization [5]. In summary, the Acting Phase is for doing the work required to reach the defined goals [6].

\section{E. Leveraging Phase}

The aim of this phase is to make the next pass through the IDEAL model more effective [5]. Based on the experiences and lessons learned from the first iteration of the IDEAL model, strategies, methods and infrastructure are evaluated, corrected and adjusted for the next passing through the cycle. The Leveraging Phase is learning from what has been done for the next iteration of the process improvement cycle [6].

\section{RELATED WORK}

The goal of the literature review was to get an overview of existing research about implementing SPI with the IDEAL model. The following keywords were used in use different combinations: software process improvement, implement IDEAL, A-SPICE, 15504, SPI, Automotive SPICE, software industry. The main sources were IEEE Xplore and Google Scholar next to proceedings of ICSE. We found 17 related papers, but none using IDEAL for implementing SPICE or A-SPICE respectively.

In this section we briefly summarize the results of six papers. Five of them studied the IDEAL model in the context of implementing CMM(I): Casey and Richardson [7], Kautz, Hansen and Thaysen [6], Drumm [8], Börjesson and Mathiassen [9], and Rasidi and Ibrahim [10]. Börjesson's and Mathiassen's study [9] reports about SPI effort at a system development center with 900 employees, the other studies examine initiating SPI in SMEs.

One, Pino, García and Piattini [11] describe the state of the art based on a systematic literature review. The authors give an overview of SPI processes in SMEs including CMM(I), ISO/IEC 15504:2004, SPICE (ISO/IEC 15504:1998), ISO/IEC 12207:2004 and ISO 9001:2000, with the application of different models (IDEAL, IMPACT and MESOPYME). They pointed out that the IDEAL model is most widely used to guide improvement [11]. They emphasize that within the Software Engineering community there is a growing concern that SEI and ISO standards are not easily applicable to small firms because they require a huge investment. Small companies cannot afford the high costs. Further, they need to adjust the process to organizational needs. They did not present results dedicated to the IDEAL model, but described some general success factors for SPI: to initiate the improvement as soon as possible but not to neglect having expert consultantship, to guide the improvement, to look for external financial aid cooperation, to involve the greatest possible number of company's employees in training, to establish an infrastructure that supports an efficient communication between the employees involved, to ensure management support and to tackle the problem of improvement from the technical perspective [11].
Kautz et al. [6] investigated the suitability of the IDEAL model for an SME to reach the specific CMM level 2. With appropriate adjustments they found the IDEAL Model suitable for SMEs [6]. As success factor they emphasize the initiation of the SPI effort as a real project with defined roles, resources and responsibilities. Further they point out the significance of education and training by external consulting, and management support [6]. They criticized the IDEAL model to distinguish between problem-centered (easily identifiable, fast fixable, short term effective) and processcentered (key process area related, long-term effective) issues not before the acting phase. Kautz et al. [6] suggest to do this already in the diagnosing phase. Further, they miss strategies to reduce the barriers in the company for software process improvement [6].

Casey and Richardson [7] explored if the IDEAL model works in a middle sized company (120 employees). They report that small enterprises tempt to disregard the Management Steering Group but advise it as a very benefit spending institution. They found that the IDEAL model was very helpful for implementing SPI successfully. In the Acting phase they claimed that the TWG's approach and available time scale did not allow or require the development of a best guess solution. Neither did it require the use of pilot testing and further refinement before implementing the improvement strategy [7].

Drumm [8] reported about the achievement of CMMI level 3 with the help of the IDEAL model. Drumm [8] exposed several points that should not be neglected: first, to involve all stakeholders before a new iteration and between phases; second, to discuss and document goals and lessons learned; third, to conduct basic training for employees at all levels; fourth, to conduct basic training for employees at every level about the software improvement process [8].

Rasidi and Ibrahim [10] researched what the success factors are when applying SPI in an IT organization. The authors conducted a case study about implementing CMM with IDEAL. The results of the paper are that management commitment and support is essential for providing the resources, monitoring of the SPI progress, prioritizing the SPI and resolving SPI issues. Furthermore, they describe that top management can be the strong motivator to people that participate in SPI [10]. They suggest that organizations with skillful employees can implement their SPI programs without outside assistance if the employees have training and internalize the knowledge of SPI processes [10]. Other important points they highlight is to manage the SPI as a real project in accordance with Kautz et al. [6].

Papers which do not review IDEAL approaches but other SPI efforts describe a few more problems that can appear while implementing SPI. Curtis [12] examined best practices and lessons learned from SPI process. He reported that problems and barriers can be the neglect of managers and people in process improvement groups about the full extent of their responsibilities implementing a successful SPI program [12]. Conradi and Dybå [13] found out that the performance of a company is closely related to the effort (person-hour) it is willing to spend on the SPI activities. Several companies dislike recruiting key technical 
employees to SPI work, because they are considered too important in ordinary business projects [13].

In summary: the IDEAL model is seen as an adequate framework for SPI processes. However, it has to be tailored and adjusted to a company, considering business objectives and resources. As a framework it offers considerable advantages for enterprises of any size. Thus, the IDEAL model based on CMM is flexible; however, it is the aim of this article to examine the suitability for A-SPICE.

\section{METHOD}

We studied the use of IDEAL for A-SPICE in its organizational context through collaborative practice research (CPR), a particular form of action research [3]. The CPR program involved close collaboration between researchers and practitioners over a six month period. Given the current state of research, we adopted a qualitative multimethod approach. At this early stage, with little empirical research in our area of interest, qualitative data were collected to explore a wide range of topics. Several methods, in particular participant observation, semi-structured interviews, and document analyses, served to validate and complement the findings. The approach we applied for data analysis was content analysis. Three researchers categorized the content of the collected data (interviews, memos, emails) independently from each other. They then compared and discussed their findings until consensus was reached.

Data collection and analyses were guided by the following research questions: 1 . Which of the IDEAL model's activities have shown to be of major importance but need to be significantly adjusted? 2 . Are there any important activities missing in the IDEAL model?

We conducted the empirical study at SMR, a global tier 1 supplier in the automotive industry with headquarters in Germany. SMR is one of the largest manufacturers of external rearview mirrors for passenger cars in the world. The company employs almost 400 practitioners at the engineering departments and approximately 6.000 people as a whole. It develops and supplies exterior mirrors, interior mirrors and camera based blind spot detection systems to nearly every carmaker in North America, South America, Europe, Asia and Australia.

The external sales in the financial year 2010/11 were approx. $€ 741$ million. SMR has secured a leading market share of more than $35 \%$ in exterior mirrors for the innovation driven European market. SMR is a subsidiary of the Samvardhana Motherson Group, an internationally operating automotive specialist for interior and exterior systems such as instrument panels, bumpers and electrical distribution systems as well as numerous polymer, elastomer and metal products.

Initially, the firm's capabilities were limited to design and development of mechanical systems, plastic molding, die-casting, electrical systems, painting and assembly. Since the late 1990s more and more electronic components and software functions have been added to the companies' product range. Today, more than 30 software experts work in the global R\&D organization, mainly in Germany and India. Their main focus is the development of intelligent camera based driver assistant systems, BUS modules and other innovative electronic systems. Depending on the specific project, SMR collaborates with various external software development partners. Software development processes in the past did not follow as high standards as demanded for example by A-SPICE, as the work was characterized by creative approaches of small teams, prototyping and applying standard principles.

Today, new products in the area of Advanced Driver Assistance Systems are being designed in an international team environment, networked with numerous customers. The parallel development of multiple product applications, modular soft- and hardware architectures as well as significantly accelerated development times demand for more systematic processes and methods. In 2011, additional software experts have been hired in India, the UK and Germany to enhance the R\&D department's capacities. As a consequence, the development processes needed to be adapted to this new software focus and elaborated in more detail to ensure a common understanding of the methods, tools and processes across countries. Moreover, customers have increasingly been requesting proof of quality with regard to processes, whereby most of them required at least A-SPICE level 2.

The company therefore decided to implement A-SPICE and a management steering group (MSG), comprised by the Executive Vice President Global Engineering and the head of the R\&D department. In July 2011, one full-time employee at the German location of the R\&D department (and co-author of this paper) was officially assigned to lead this SPI effort as A-SPICE project manager. Technical advice was given by the A-SPICE consulting group, consisting of one quality manager (certified ISO/IEC 15504 Provisional Assessor, Automotive SPICE®), one process engineer at the mechanics department, and the head of the R\&D India department.

The assessment of the organization's climate showed that the majority of practitioners at the $R \& D$ department did recognize the need for the elaboration of a detailed development process. Due to their experiences in the past, they were aware of the SPI benefits in terms of clear roles and responsibilities, standard templates and process guidelines.

The A-SPICE project manager set up the SPI project manual, which contained the project scope, life cycle, work breakdown structure, estimation of main parameters, risk management plan, staffing plan, interfaces as well as tools and processes. It was decided to implement the HIS scope and achieve level 2 as middle-term goal (to be completed by the end of 2012). The number and type of pilot projects per SPICE level was also defined. Moreover, the formation of 15 technical working groups (TWGs), all located in the German headquarters, was envisaged to address the 15 A-SPICE process areas. This Initiating Phase lasted until August 2011, when the MSG approved the project manual.

During the Diagnosing Phase, the A-SPICE project manager first of all had nine individual meetings with the TWG leaders and some of the TWG members. She explained the requirements of the specific A-SPICE process area and 
asked about current practices and related documents. Gaps between the "as-is" state and "to-be" state were documented by the project manager and then presented to all TWG members and the head of the R\&D department in separate meetings depending on the topic. The findings were discussed and a consensus about the as-is-state as well as the next steps was reached. This phase ended in October 2011.

Subsequently, the project manager finalized the A-SPICE action plan with a description of the deliverables and timelines (Establishing Phase). Phase four of the IDEAL model, the Acting Phase, started in November 2011 and is still ongoing. So far, data collection and analysis covered the IDEAL subphases 1.1 (getting started) until 4.3 (pilot potential solutions), thus including 33 of a total of 47 subphases.

Since the Initiating Phase, a research collaboration has been established between the A-SPICE project manager and a university research group. A life cycle model, the IDEAL model, was jointly selected to guide the company through its SPI efforts. We used data and methodological triangulation [14]. The research group conducted semistructured interviews with the project manager and three TWG leaders once a month to reflect about the suitability of the model and general barriers and leverage points. In addition, several documents that illustrated the current practices were analyzed (e.g. requirement specifications, test plans, project manuals, traceability matrices).

Following Baskerville and Wood-Harper's [15] framework the action research can be characterized as follows. The process was iterative, involving repeating sequence of activities. The structural guidance was rigorous that is characterized by delineated stages, steps and activities carried out in a sequence. And, third, the researcher's involvement was collaborative, meaning that the researchers are equal co-workers with the study subjects. The dual purpose was organizational development and scientific knowledge.

\section{RESULTS}

In the following sections, we address the research question whether some of the IDEAL model's activities have shown to be of major importance but needed to be significantly adjusted to the specific context. We summarize the findings according to topic, and not by phase, as several activities of the IDEAL model are more or less repeated in the five phases.

\section{A. Participation}

\section{1) Infrastructure}

The Technical working groups (TWGs), formed in the Establishing Phase, represent one of the main components of the SPI infrastructure in the IDEAL model. Their purpose is to develop solutions for a specific area of the SPI program. Our data indicate that adjustments are needed with regard to the TWGs' tasks and the time of formation. It is advisable to form TWGs as early as in the Initiating Phase. Due to the fact that the main areas of improvement are known from the beginning when implementing A-SPICE, TWGs can already make contributions in the Diagnosing Phase. However, our results suggest the role of the TWGs to be redefined. The active development of solutions on their own as far as possible, although claimed as critical success factor due to the participative aspect, was not a workable solution in the present study. Most practitioners at the R\&D department neither had sufficient expertise nor interest to "translate" abstract A-SPICE standards into practical solutions. Without external training, it would have been impossible to interpret the standards correctly. To train all members of the working groups would have been costly and time-consuming. Moreover, the abstract language deterred the practitioners and was not very compatible with their daily practical work. Thus, the A-SPICE project manager had to develop potential solutions and then present them to the TWGs to get feedback. This person was then, instead of the TWGs, directly reporting to the process owners of the MSG. Furthermore, due to the overlapping A-SPICE process areas and base practices, it seemed to make more sense to have one person being in charge of the entire implementation and coordinating solutions than forming 15 working groups that have to interact with each other in order to adjust their approaches and solutions.

\section{2) Definition of SPI goals}

A further participative aspect is emphasized in the following IDEAL activity: "Support is built by involving the people affected by the program in the early, defining parts of the program when they can more easily make a difference and increase their stake in the outcomes" [5, p. 26]. This statement seems to be correct in the context of general SPI programs. However, participation is again limited with regard to defining the SPI goals when implementing ASPICE. "What" has to be achieved is already defined by the ISO standard, only "how" it will be achieved can be decided by the company. Not even the prioritization of the improvement areas can significantly be modified, because all 15 process areas have to be covered (from the lowest ASPICE level onwards). Merely the chronological order within one cycle can be defined. In our empirical study, for example, many practitioners expressed the need for improving the requirements engineering process (by introducing the tool DOORS and a more formal change process $[16 ; 17])$. This is what was given first priority in the SPI effort.

Also, a way to enable participation of employees affected by the program is to involve them in the decision-making process about the question whether only the requirements of A-SPICE level 1 should be implemented in the first cycle or parts of higher levels as well. In our research study, some practitioners stated that a detailed definition of all A-SPICErelevant project types as well as of all general project roles (including A-SPICE relevant tasks and guidelines) at the $R \& D$ department would enhance their understanding of the SPI effort. This is part of A-SPICE level 3. The SPI project manager assigned high priority to this request in the first cycle in order to maintain motivation.

\section{B. Communication}

The IDEAL guide recommends communicating to the entire organization about what the SPI team is "learning and 
what is happening" [5, p.14]. More specifically, it is described in the Diagnosing Phase that to ,all members of the organization the same message" has to be communicated concerning the results of the baselining activities [5, p.66]. However, the findings of this study suggest limiting the size of the audience to only those employees whose work is directly related to the A-SPICE process area to be talked about. For example, we would not recommend explaining to software developers the details of activities with regard to high-level change management, where only project managers as well as middle and senior management are actively involved. In general, A-SPICE requirements are very finegrained - so are the baselines, templates and work instructions. Not every employee has the time, knowledge and interest for it and it can be counterproductive to talk about topics that parts of the audience deem irrelevant for themselves. Moreover, communicating in detail all the baselining results could trigger criticism against colleagues. This can negatively affect the authority, acceptance and perceived competence of others. Finally, and most importantly, as long as the solutions are not tested in a pilot project, it seems to be counterproductive to present them to a broad audience at this early stage. Of course, brief updates to everybody about the general SPI progress are helpful to maintain motivation.

\section{Prioritization}

When implementing A-SPICE, the IDEAL activity about deciding "which of the many competing SPI recommendations and actions will be launched and funded" $[5$, p. 86] played a minor role. The company SMR needed to be able to demonstrate a well-documented process and work practices in all 15 SPICE process areas (HIS scope). More specific guidelines about the prioritization of these SPICE areas would be helpful. In the empirical study, the process fields "quality assurance" and "system requirements analysis" were prioritized. This approach has shown to be successful, as these areas build the basis for other process fields. For example, quality assurance determines critical baselines, reviews and quality gates that have to be included into the project and configuration management plan. Furthermore, a sound system requirements analysis is of uttermost importance for the subsequent processes (e.g. system and software architectural design, software construction, and system testing).

\section{Management Commitment}

The IDEAL model stresses the importance of management commitment, which was confirmed by the results of our study. The related tasks described in the model, however, seemed to be mainly limited to the active involvement of the management in the planning activities and allocation of resources. More concrete and diverse strategies on how to maintain the commitment and make it visible would be helpful. In the present study, the head of the R\&D department (HOD) frequently asked about the SPI progress in the weekly department meetings, and showed interest as well as support. Another strategy was to put ASPICE posters on the wall of the HOD's office and of the main departmental meeting room. At the high management level, management commitment was ensured through the inclusion of the strategic goal "continuous process improvement for software intensive systems" into the Quality Management Manual and Quality Management Review.

\section{E. Information Sharing}

Although there was only one software division at SMR, the R\&D department, and thus only one A-SPICE implementation program, several parallel improvement efforts about the general product engineering process existed. This was part of the continuous improvement program of the firm. Several minor local improvement initiatives were distributed across the globe. The IDEAL model addressed this situation by recommending the facilitation and encouragement of information sharing through periodic formal meetings in order to not reinvent the wheel. In our study, however, process improvements were primarily a small part of the individuals' work. Meetings did take place, but not periodically. Informal personal networks proved to be invaluable for information sharing. Even if some groups set up meetings for exchanging information, it was often not communicated through formal mechanisms. The A-SPICE project manager usually obtained information about process meetings through networking skills rather than formal invitations. The IDEAL model does take into consideration the importance of informal "support networks" [5, p. 45]. It describes the planning of team building activities in order to facilitate this success factor. In the present study, the recommended mechanisms such as common training and collaboration on assessments were often not applicable due to the small number of people and distributed locations. Personal networking skills therefore became more important and should be included in the IDEAL model.

In the following sections, we address the research question whether there are any important activities missing in the IDEAL model.

\section{F. Informal Networks between Companies}

Based on our experiences in the context of the present study, external training on A-SPICE at consulting companies is helpful to a certain limit. It is appropriate to develop a basic understanding of A-SPICE requirements, i.e. to be able to interpret the standards correctly. Nevertheless, the concrete realization is usually not described in detail in the training and still represents a challenge for SPI practitioners. In our study, the SPI project manager benefited significantly from the informal exchange of experiences with SPI managers of other companies in the automotive industry that had $R \& D$ departments of a similar size.

\section{G. Usability}

Our findings suggest that user-friendliness of the templates and newly introduced tools plays an important role for the sustainable implementation of A-SPICE. Due to the very detailed requirements of A-SPICE, it can be tempting to develop quite complex templates in order to ensure that audits will be passed smoothly. The usage of specialized 
tools has sometimes a similar function. However, clear and simple templates and guidelines for each project role as well as a limited number of tools can be critical so that practitioners are not overwhelmed by all the changes. Even if the company decided to implement A-SPICE level 3 in the long run, not too many new and complex tools should be introduced at the beginning.

\section{H. Early Decision-Making}

As mentioned above, some requirements of A-SPICE level 2 or 3 were already implemented in the first cycle of the SPI effort at SMR. Although detailed, tool-supported process descriptions and tailoring guidelines are part of level 3 , it fostered the orientation and motivation of the practitioners. This shows that early decision-making about which A-SPICE level to achieve as a long-term goal can be of central importance.

\section{DISCUSSION}

Overall, our findings indicate that many tasks described in the IDEAL model contributed to the present success of the A-SPICE implementation effort at SMR. That is, the suitability of the IDEAL model for the implementation of ASPICE in medium-sized R\&D departments is high. This result is in line with research on the implementation of CMM(I) in SMEs $[6 ; 7]$. However, some of the recommended activities needed to be adjusted, especially with regard to the factors participation and communication. Furthermore, informal networks between companies, early decision-making and usability are shown to play an important role and might be added to the IDEAL model. These findings on success factors to be adjusted or added have not been discussed in previous studies on the IDEAL model $[6 ; 7 ; 8 ; 9 ; 10]$ and have both theoretical and practical implications. They add to the body of knowledge on SPI and success factors [12]. Furthermore, companies that are planning to implement A-SPICE can immediately draw upon our concrete suggestions about how to use the adapted IDEAL model.

This study has the following limitations: Data collection and analysis covered the IDEAL subphases 1.1 (getting started) until 4.3 (pilot potential solutions). Phases four to five are still to be completed. Furthermore, the influence of the organizational and departmental culture on our results could not be controlled.

Future work should further refine and validate our findings. A quantitative study would help to examine the relative importance of the newly added factors and adjustments we proposed. Also, future studies should investigate to what extent A-SPICE helps SPI programs to survive and be more efficient. Certain success factors of the IDEAL model are about survival of the program (e.g. "maintain visibility") and selection of goals, but did not seem to be critical in the context of the present study, as the certification of a specific A-SPICE level is often part of the customer requirements in development projects.

\section{REFERENCES}

[1] Höhn, H., "Software-Engineering nach Automotive SPICE," 1 ed., Heidelberg: Dpunkt-Verl. 2009.

[2] Iversen, J.H., Mathiassen, L., and Nielsen, P.A., "Managing Risk in Software Process Improvement: An Action Research Approach." MIS Quarterly, vol. 28(3), 2004, pp. 395-433.

[3] Runeson, P. and Höst, M., "Guidelines for conducting and reporting case study research in software engineering." Empirical Software Engineering, vol. 14(2), 2009, pp. 131-164.

[4] HIS 2011. HIS - Hersteller Initiative Software - HIS, http://www.automotive-his.de/.

[5] McFeeley, B., "IDEAL: A User's Guide for Software Process Improvement," Ft. Belvoir: Defense Technical Information Center 1996.

[6] Kautz, K., Hansen, H.W., and Thaysen, K., "Applying and adjusting a software process improvement model in practice: the use of the IDEAL model in a small software enterprise," Proc., ACM, 2000, pp. 626-633.

[7] Casey, V. and Richardson, I., "A practical application of the IDEAL model." Software Process: Improvement and Practice, vol. 9(3), 2004, pp. 123-132.

[8] Drumm, P.M., "SW Process Improvement (Example) - CMMI and IDEAL," Enterim LLC 2009.

[9] Borjesson, A. and Mathiassen, L., "Making SPI happen: the IDEAL distribution of effort," Proc. Proceedings of the 36th Hawaii International Conference on System Sciences (HICSS’03), 2003, pp. 10 pp.

[10] Rasidi, H. and Ibrahim, R., "The Adoption of Software Process Improvement (SPI) Program in the Construction Industry." ALAM CIPTA, International Journal on Sustainable Tropical Design Research \& Practice, vol. 3(1), 2008, pp. 79-90.

[11] Pino, F.J., García, F., and Piattini, M., "Software process improvement in small and medium software enterprises: a systematic review." Software Quality Journal, vol. 16(2), 2008, pp. 237-261.

[12] Curtis, B., "Software process improvement: best practices and lessons learned," Proc. ICSE, 2000, pp. 4-11.

[13] Conradi, R., Dyba, T., Sjoberg, D.I.K., and Ulsund, T., "Lessons learned and recommendations from two large norwegian SPI programmes." Lecture notes in computer science, 2003, pp. 32-45.

[14] Stake, R.E., "The art of case study research," Sage Publications, Inc 1995.

[15] Baskerville, R. and Wood-Harper, A.T., "Diversity in information systems action research methods." European Journal of Information Systems, vol. 7(2), 1998, pp. 90-107.

[16] Berkovich, M., Esch, S., Leimeister, J.M., and Krcmar, H., "Requirements engineering for hybrid products as bundles of hardware, software and service elements-a literature review." Proceedings of Wirtschaftsinformatik 2009, pp. 727-736.

[17] Berkovich, M., Leimeister, J., and Krcmar, H., "Requirements Engineering for Product Service Systems." Business \& Information Systems Engineering, vol. 3(6), 2011, pp. 369-380. 